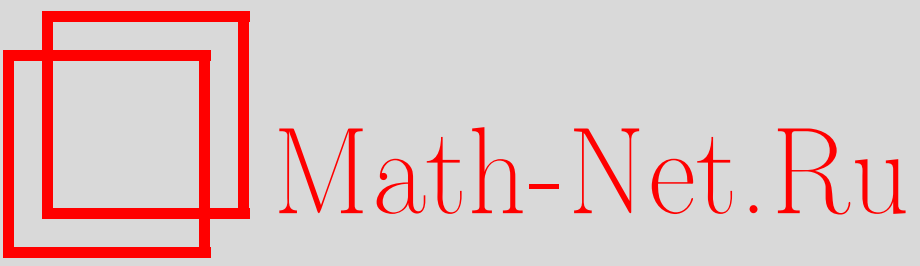

А. Э. Гутерман, Преобразования неотрицательных целочисленных матриц, сохраняющие определитель, УМН, 2003, том 58, выпуск 6, 147-148

DOI: https://doi.org/10.4213/rm680

Использование Общероссийского математического портала Math-Net.Ru подразумевает, что вы прочитали и согласны с пользовательским соглашением

http://www.mathnet.ru/rus/agreement

Параметры загрузки:

IP: 35.174 .16 .151

26 апреля 2023 г., 08:09:04 


\section{ПРЕОБРАЗОВАНИЯ НЕОТРИЦАТЕЛЬНЫХ ЦЕЛОЧИСЛЕННЫХ МАТРИЦ, СОХРАНЯЮЩИЕ ОПРЕДЕЛИТЕЛЬ}

\section{А.Э. ГУТЕРМАН}

Теория линейных отображений, сохраняющих матричные инварианты, берет свое начало с работы Фробениуса [1], где охарактеризованы линейные биективные отображения матриц над полем комплексных чисел, сохраняющие определитель. В дальнейшем эта область активно развивалась, в частности, были получены аналоги теоремы Фробениуса для матриц над различными полями и кольцами, см. [2], [3]. Вместе с тем, в связи с многочисленными приложениями в комбинаторике, теории графов и теории информации, постоянно растет интерес к линейной алгебре над полукольцами, см. [4]. В том числе интенсивно ведутся исследования линейных отображений, сохраняющих матричные инварианты над полукольцами, см. [3; гл. 9]. Однако общий вопрос об аналоге теоремы Фробениуса для матриц с коэффициентами из полукольца остается открытым. В настоящей работе получен аналог теоремы Фробениуса для матриц с неотрицательными целыми коэффициентами, а именно, охарактеризованы аддитивные биективные отображения, сохраняющие определитель. Кроме того, охарактеризованы аддитивные биек тивные отображения, сохраняющие перманент и другие коэффициенты ладейного многочлена.

Пусть $\mathscr{M}_{n}\left(\mathbb{Z}_{+}\right)$обозначает множество всех $(n \times n)$-матриц с неотрицательными цельми коэфофициентами, $E_{i j}$ - матричная единица, $C_{i}$ - матрица с единицами в $i$-м столбце и нулями во всех остальных столбцах, $R_{j}$ - матрица с единицами в $j$-й строке и всеми осталшными элементами, равными нулю.

ОПРЕДЕЛЕниЕ 1 . Отображение $T: \mathscr{M}_{n}\left(\mathbb{Z}_{+}\right) \rightarrow \mathscr{M}_{n}\left(\mathbb{Z}_{+}\right)$назьвается перестановочным, если существуют матрицы перестановки $P, Q \in \mathscr{M}_{n}\left(\mathbb{Z}_{+}\right)$такие, что $T(X)=P X Q$ для всех $X \in \mathscr{M}_{n}\left(\mathbb{Z}_{+}\right)$или $T(X)=P X^{t} Q$ для всех $X \in \mathscr{M}_{n}\left(\mathbb{Z}_{+}\right)$.

Лемма 1. Пусть $T: \mathscr{M}_{n}\left(\mathbb{Z}_{+}\right) \rightarrow \mathscr{M}_{n}\left(\mathbb{Z}_{+}\right)$- линейное отображсние, заданное как $T\left(E_{i j}\right)=E_{\sigma(i, j)}$ для всех $(i, j)$, где $\sigma$ - перестановка на множестве пар $\{(i, j)$ । $1 \leqslant i \leqslant n, 1 \leqslant j \leqslant n\}$. Если отображение $T$ сохраняет определитель, то $T$ - перестановочное отображение, причем перестановки, соответствующие матрицам $P$ и $Q$ имеют одинаковую четность.

ДокАЗАТЕЛЬСтво. Покажем, что $T^{-1}$ отображает строки матрицы в строки или столбцы. Допустим противное, т.е. что существуют такие индексы $i, j, k$, что $T^{-1}\left(E_{i j}\right)$ и $T^{-1}\left(E_{i k}\right)$ не лежат в одной строке или в одном столбце. Тогда существуют матричные единицы $E_{i_{1} j_{1}}, \ldots$, $E_{i_{n-2} j_{n-2}}$ такие, что матрица $A=T^{-1}\left(E_{i j}\right)+T^{-1}\left(E_{i k}\right)+E_{i_{1} j_{1}}+\cdots+E_{i_{n-2} j_{n-2}}$ является матрицей перестановки. В этом случае $\operatorname{det} A= \pm 1$. Отсюда $\operatorname{det} T(A)= \pm 1$. С другой стороны, $T(A)=E_{i j}+E_{i k}+T\left(E_{i_{1} j_{1}}\right)+\cdots+T\left(E_{i_{n-2} j_{n-2}}\right)$. По условию матрица $T\left(E_{r s}\right)$ имеет в точности один ненулевой элемент для всех $r, s=1, \ldots, n$. Посколшку $E_{i j}$ и $E_{i k}$ лежат в одной строке, из принципа Дирихле следует, что матрица $T(A)$ имеет нулевую строку. Следовательно, $\operatorname{det} T(A)=0$. Полученное противоречие показьвает, что $T^{-1}$ переводит элементы матрицы, лежащие в одной в строке, в элементы, лежащие в одной строке или одном столбце. Аналогично, легко видеть, что $T^{-1}$ переводит столбцы в строки или столбцы. Следовательно, то же самое верно и для отобажения $T$.

Поскольку ни одна комбинация $k$ строк и $l$ столбцов, где $k+l=n$, не может покрыть матрицу $J=\sum_{i, j=1, \ldots, n} E_{i j}$, за исключением случая $l=0$ или $k=0$, получаем, что или образ при отображении $T$ каждой строки есть строка, а столбца - столбец, или образ каждой строки столбец, а столбца - строка. Следователњно, существуют такие матрицы перестановки $P$ и $Q$, что $T\left(R_{i}\right)=P R_{i} Q$ и $T\left(C_{j}\right)=P C_{j} Q$ для всех $i, j$ или $T\left(R_{i}\right)=P\left(R_{i}\right)^{t} Q$ и $T\left(C_{j}\right)=P\left(C_{j}\right)^{t} Q$ для всех $i, j$. Отсюда следует, что $T\left(E_{i j}\right)=P E_{i j} Q$ для всех $i, j$ или $T\left(E_{i j}\right)=P E_{j i} Q$ для всех $i, j$, т.е. $T(X)=P X Q$ для всех $X \in \mathscr{M}_{n}\left(\mathbb{Z}_{+}\right)$или $T(X)=P X^{t} Q$ для всех $X \in \mathscr{M}_{n}\left(\mathbb{Z}_{+}\right)$.

Работа выполнена при частичной поддержке гранта Президента РФ "Ведущие научные школы”. 
Наконец, поскольку отображение $X \mapsto P X Q\left(X \mapsto P X^{t} Q\right)$ должно сохранять определитель, имеем: $\operatorname{det}(P Q)=\operatorname{det}(P E Q)=\operatorname{det} E=1$, где $E=\sum_{i=1}^{n} E_{i i}$ - единичная матрица. Отсюда следует, что матрицы $P$ и $Q$ отвечают перестановкам одинаковой четности.

ОПредЕлениЕ 2. Ненулевая матрица $A \in \mathscr{M}_{n}\left(\mathbb{Z}_{+}\right)$называется неразложимой, если для любых матриц $X, Y \in \mathscr{M}_{n}\left(\mathbb{Z}_{+}\right)$из $X+Y=A$ следует, что или $X=0$, или $Y=0$.

Теорема 1. Пусть $T: \mathscr{M}_{n}\left(\mathbb{Z}_{+}\right) \rightarrow \mathscr{M}_{n}\left(\mathbb{Z}_{+}\right)$- аддитивное биективное отображсение. Отобрахсение $T$ сохраняет определитель в том и только том случае, когда $T$ - перестановочное отображение, причем перестановки, соответствующие матрицам $P$ $u Q$, имеют одинаковую четность.

ДокАЗАТЕльство. Легко видеть, что отображения описанного типа сохраняют определитель. Заметим, что автоморфизмы аддитивного моноида $\mathscr{M}_{n}\left(\mathbb{Z}_{+}\right)$переводят неразложимые элементы в неразложимые и единственными неразложимыми элементами в $\mathscr{M}_{n}\left(\mathbb{Z}_{+}\right)$являются матрицы $E_{i j}, 1 \leqslant i, j \leqslant n$. Отсюда следует, что $T\left(E_{i j}\right)=E_{\sigma(i, j)}$ для всех $(i, j)$ для некоторой перестановки $\sigma$. Следовательно, по лемме 1 отображение $T$ имеет требуемый вид.

ОПРЕДЕЛЕниЕ 3 . Пусть матрица $A \in \mathscr{M}_{n}\left(\mathbb{Z}_{+}\right)$. Многочлен $R_{A}(x)=\sum_{i=0}^{n} p_{i} x^{i} \in \mathbb{Z}[x]$, где коэффициент $p_{0}=1$, а при $1 \leqslant i \leqslant n$ коэффициент $p_{i}$ равняется сумме перманентов всех $(i \times i)$-подматриц в $A$, назьвается ладейным многочленом матрицы $A$.

Легко видеть, что, в отличие от определителя, перманент и другие коэффициенты ладейного многочлена остаются неизменными при всех перестановочных преобразованиях. В работе [5] было показано, что над некоторыми полукольцами линейнье отображения, сохраняющие перманент (старший коэффициент ладейного многочлена), оставляют неизменными все коэффициенты ладейного многочлена, и установлено, что соответствующие преобразования исчерпываются перестановочными отображениями. Предлагаемый метод позволяет ослабить условия теоремы из [5] и получить характеризацию отображений, сохраняющих любой коэффициент ладейного многочлена, в частности, доказать следующий резултат:

Tеорема 2. Рассмотрим аддитивное биективное отображение $T: \mathscr{M}_{n}\left(\mathbb{Z}_{+}\right) \rightarrow$ $\mathscr{M}_{n}\left(\mathbb{Z}_{+}\right)$и фиксированное челое число $i, 2 \leqslant i \leqslant n$. Отображсение $T$ сохраняет $i$-й коәффициент ладейного многочлена в том и только том случае, когда T перестановочное отображение.

При $i=n$ получаем утверждение теоремы из [5].

\section{СПИСОК ЛИТЕРАТУРЫ}

[1] G. Frobenius // Über die Darstellung der endlichen Gruppen durch lineare Substitutionen, Sitzungsber. Berlin: Preuss. Akad. Wiss. Berlin, 1897. P. 994-1015. [2] A. Guterman // Linear and Multilinear Algebra. 2001. V. 48. № 4. P. 293-311. [3] C.-K. Li, S. Pierce, N.-K. Tsing (Eds.). A Survey of Linear Preserver Problems. Taylor \& Francis, 1992. (Linear and Multilinear Algebra. V. 33. № 1-2 (Special issues).) [4] K. Głazek. A Guide to the Literature on Semirings and their Applications in Mathematics and Information Sciences. Dordrecht: Kluwer, 2002. [5] L. B. Beasley, N. J. Pullman // Linear Algebra Appl. 1987. V. 90. P. 33-46. 